\title{
Implementation of Accompanying Family Management for Gastroenterology Patients During the COVID-19 Epidemic
}

\author{
Guiyan Wen ${ }^{1,2}$, Jinglan Luo ${ }^{1,2,}$, , Shaohui Tang ${ }^{1,2}$ \\ ${ }^{1}$ Department of Gastroenterology, the First Affiliated Hospital of Jinan University, Guangzhou, China \\ ${ }^{2}$ School of Nursing, Jinan University, Guangzhou, China
}

Email address:

wengy78@126.com (Guiyan Wen), tljl@jnu.edu.cn (Jinglan Luo)

${ }^{*}$ Corresponding author

To cite this article:

Guiyan Wen, Jinglan Luo, Shaohui Tang. Implementation of Accompanying Family Management for Gastroenterology Patients During the COVID-19 Epidemic. American Journal of Nursing Science. Vol. 9, No. 6, 2020, pp. 450-454. doi: 10.11648/j.ajns.20200906.22

Received: December 2, 2020; Accepted: December 14, 2020; Published: December 22, 2020

\begin{abstract}
The objective of this study is to explore the effect of implementing accompanying family management in gastroenterology patients during the COVID-19 epidemic. According to the guidelines for the prevention and control of COVID-19 issued by the National Health Commission and the Guangdong Provincial Health Commission, as well as the First Affiliated Hospital of Jinan University and patients' demands, we implemented an accompanying family management system for gastroenterology patients. The management plan not only listed the permission criteria for accompanying family, but also offered escorts with COVID-19 nucleic acid testing, COVID-19 case screening, temperature measurement, symptom observation, health education, and health protection. The results of this study revealed the following main findings: From January 23 to April 23, 2020, a total of 438 inpatients and 113 family escorts were admitted to the hospital, who demonstrated $100 \%$ compliance with epidemic prevention and control measures, no infections, and an average score of 96.2 in the patient satisfaction survey. It can be concluded that the establishment and strict implementation of an accompanying family management system together with good epidemic prevention and control practices not only substantially reduces the accompanying family rate without compromising the quality and safety of patient care, but also effectively prevents in-hospital infections.
\end{abstract}

Keywords: COVID-19, Accompanying Family, Management Implementation, Epidemic Prevention and Control

\section{Introduction}

Cases of COVID-19 were first reported in December 2019 in China [1]. On January 20, 2020, the National Health Commission of the People's Republic of China issued Announcement No. 1, which proposed that as an acute respiratory infectious disease, COVID-19 was defined as a category B infectious disease under the Law of the People's Republic of China on the Prevention and Control of Infectious Diseases, and should be managed as a category A infectious disease [2]. It mainly transmits through respiratory droplets and close contact, with the general population as its susceptible crowd. Reports from existing cases have suggested that although most of the patients demonstrate a good prognosis, a few patients can progress to a critical condition. In addition, the elderly and those with chronic underlying diseases often have a poor prognosis, making national epidemic prevention and control important. The Department of Gastroenterology of the First Affiliated Hospital of Jinan University treated 438 non-COVID-19 patients with digestive system diseases during the epidemic spanning the Spring Festival. Of these patients, $61.44 \%$ were in a severe or critical condition, $5.51 \%$ were critically ill, and $55.93 \%$ were severely ill. Most patients also suffered from other diseases, lacked daily living abilities, and had low body resistances, thereby requiring significant accompanying family and psychological support. Accompanying family refers to the completion of inpatients' daily care by family members. It not only meets the needs of inpatients and their families and makes patients feel more secure, but also encourages them to actively cooperate with treatment, improves their psychological state, and promotes their recovery [3]. The technical guidelines on the prevention and 
control of COVID-19 in medical institutions issued by the National Health Commission has suggested that it is important to strictly carry out the prevention and control of COVID-19 infections, so as to minimize the risk of transmission in medical institutions and ensure the quality and safety of medical care [4]. To reduce the risk of transmission within hospitals, in addition to the protection of medical personnel, the management of patients and escorts should be strengthened, crowding in the hospital should be reduced, and infections from external sources should be prevented. The effective prevention and control of nosocomial infections is a key measure to prevent the spread of COVID-19. However, due to the strong infectivity of this novel virus, the efficacy of previous nosocomial infection prevention and control measures remains questionable. To meet inpatients' needs of care and companionship and prevent them from being infected with COVID-19 without compromising their quality of care, based on relevant prevention and control guidelines issued by the National Health Commission, the Department of Gastroenterology of the First Affiliated Hospital of Jinan University implemented an accompanying family management system during the epidemic, which achieved superior outcomes. The purpose of this paper is to report the implementation and outcome of this system.

\section{Establishment of the Accompanying Family Management System}

Based on the hospital's patient admission procedure and escort and visit management requirements for the prevention and control of COVID-19, doctors and nurses of the Department of Gastroenterology established a semi-closed accompanying family management system, which determined whether an escort was allowed based on the patient's condition, mental status, and daily living abilities. Major contents of the system include: (1) Doctors and nurses jointly assess whether the patient needs to have an escort. A family escort is allowed when one or more of the following criteria are met: patient is severely or critically ill, patient's daily living ability score $\leq 40$, or patient's mental health questionnaire result is positive. (2) A "Notification on the prevention and control of COVID-19" is created. (3) "Patient accompany management measures" and "Patient accompany management rules" are formulated. (4) Care contents of the escort are clarified. (5) A throat swab sample must be acquired from the escort for COVID-19 nucleic acid testing, and entrance is allowed only when the result is negative.

\section{Implementation of the Accompanying Family Management System}

\subsection{Assessment of Patients on Admission}

(1) Doctors will assess the patient's condition and control the number of admissions. The admission of critically ill and emergency patients is prioritized over other patients. In addition, patients should have completed COVID-19 screening prior to admission, including taking a COVID-19 nucleic acid test using throat swabs, completing a COVID-19 epidemiological questionnaire, measuring body temperature, and taking a chest $\mathrm{CT}$ examination as well as routine blood tests. (2) Whether the patient needs to be accompanied is assessed based on their condition, daily living abilities, and mental health. Accompanying family are allowed when one or more of the following criteria are met: patient is severely or critically ill, patient's daily living ability score $\leq 40$, or patient's mental health questionnaire result is positive.

\subsection{Bed Arrangement}

Patients who are allowed accompanying family are admitted in single rooms when possible. When there are insufficient single rooms, an empty bed is used to separate two patients to reduce the number of people in the ward.

\subsection{Health Education}

Nurses will distribute "Notification on the prevention and control of COVID-19" and "Patient accompaniment management rules" to patients and escorts. Relevant infection control knowledge is also distributed through WeChat. In addition, the ward TV is turned on to guide patients and escorts to watch "Fighting the epidemic" news and special reports. Alternatively, the accompanying family management system during the epidemic is demonstrated to patients and escorts by sending "Hospitalization instructions" via a cloud follow-up information platform or oral description by nurses. Responsible nurses are also required to explain the necessity of mask wearing and hand hygiene to patients and escorts and demonstrate the correct method of putting on masks and washing hands. During the epidemic, a total of 438 patients and 113 escorts were trained, who achieved a 100\% compliance rate in mask wearing and hand hygiene. Lastly, the content of accompanying family is clarified, which includes daily life care, such as assisting the patient in cleaning, eating, bathing, changing clothes, going to the toilet, carrying out physical activities, as well as providing mental support and accompanying the patient to complete examinations.

\subsection{Strict Management of Accompanying Family}

(1) The number of escorts is strictly limited according to the patient's needs, and only one family member is allowed for each patient. During hospitalization, the escort enters or leaves the ward with their escort card and cannot leave the hospital area without permission from doctors or nurses. The replacement of an escort is only permitted after assessment and approval by both doctors and nurses. (2) Patients are encouraged to replace family escorts with full-time hospital escorts. Both doctors and nurses are required to encourage family members to hire hospital escorts to look after the patient, the hospital after life care training escort personnel, its work includes escort post responsibilities, work code and 
service honorific, taboo language and other content [5]. Escort nursing process safety, defects also significantly reduced [6]. Relatively fixed, 1 escort care 2 6 patients, assist nurses to provide life care for patients. Meeting the physiological needs of patients is primary focus of nursing work [7]. During the epidemic, instead of accompanying family, 159 patients were looked after by four full-time hospital escorts, thereby reducing the number of escorts in the hospital.

\subsection{Implementation of Accompanying Family Management}

(1) Symptom observation: The body temperature of escorts is measured daily at 10:00 and 16:00. The detection of fever or suspicious respiratory and digestive tract symptoms in any escort is reported to the chief nurse, the department director, and the health care department. The escort is then directed to the fever clinic, and their accompanying right is cancelled. (2) Environmental management: Each ward is equipped with a rapid hand disinfection station. The windows of the ward are opened for ventilation at least twice a day, each with a duration of at least 30 minutes. The floor of the ward and the bathroom is mopped twice a day with $500 \mathrm{mg} / \mathrm{L}$ chlorinated disinfectant. Toilets, sinks, and walls are cleaned at least once a day with $500 \mathrm{mg} / \mathrm{L}$ chlorinated disinfectant. Each ward is assigned one towel, replaced immediately after usage. Reuse of the towel after soaking is strictly prohibited. (3) Personal protection: Escorts are required to always wear masks and wash hands before and after contact with patients. When escorts return to the ward with patients after examinations, nurses must check their escort cards and instruct them to wash hands before entering. In addition, escorts are prohibited from leaving the hospital area or entering the office area to inquire about the patient's condition. (4) Temporary visit: If the patient is critically ill or dying, the doctor in charge can issue a "temporary visit certificate," which allows the patient's family member to enter the ward. Only one person is allowed to visit once a day for a duration of 30 minutes. (5) Meal management: Escorts must order meals from the hospital's canteen rather than from outside. (6) The responsible nurse is required to assist the escort in looking after the patient's daily life, such as cleaning, eating, bathing, changing clothes, going to the toilet, and conducting physical activities, while ensuring the patient's safety. In addition, under the nurse's guidance, the escort should provide the patient with mental support such as comforting them and encouraging them to actively engage in treatment. Lastly, the escort should make efforts to increase the patient's contentment and reduce the risk of falling, thereby eliminating the patient's worries and fears and improving their confidence and cooperation in diagnosis and treatment.

\section{Results}

During the epidemic prevention and control period from January 23, 2020 to April 23, 2020, the accompanying family management system was introduced to a total of 438 inpatients in the Department of Gastroenterology, from which 277 patients were deemed eligible for family accompany. There were a total of 113 family escorts and 2 professional escorts, resulting in an accompanying family rate of $25.78 \%$, which was successfully lowered compared to before. In addition, the average patient satisfaction score was 96.2, and the compliance rate of both the patients and the escorts were $100 \%$. The implementation of the accompanying family management system successfully prevented COVID-19 infections from external sources, so that there were zero infections among the medical personnel, patients, and escorts in the department. During the implementation, a total of 277 patients were identified as suitable for family accompaniment, details of which are listed in Table 1.

Table 1. Case analysis of accompanying family.

\begin{tabular}{|c|c|c|c|c|c|}
\hline Accompanying family criteria that are met & $\begin{array}{l}\text { No. of } \\
\operatorname{cases}(\mathbf{n})\end{array}$ & $\begin{array}{l}\text { Actual No. of } \\
\text { family escorts (n) }\end{array}$ & $\begin{array}{l}\text { Accompanying } \\
\text { family rate }(\%)\end{array}$ & $\begin{array}{l}\text { No. of cases looked after by } \\
\text { two professional escorts (n) }\end{array}$ & $\begin{array}{l}\text { No. of cases } \\
\text { without escorts (n) }\end{array}$ \\
\hline Only meeting (1) Patients who are critically ill & 212 & 67 & 31.60 & 53 & 92 \\
\hline Only meeting (2) Patients with a daily living score $\leq 40$ & 4 & 2 & 50.00 & 2 & 0 \\
\hline $\begin{array}{l}\text { Only meeting ( } 3 \text { ) Patients whose mental health } \\
\text { questionnaire result is positive }\end{array}$ & 4 & 1 & 0.33 & 0 & 3 \\
\hline Meeting both (1) and (2) & 49 & 38 & 77.55 & 11 & 0 \\
\hline Meeting both (1) and (3) & 8 & 5 & 62.50 & 3 & 0 \\
\hline
\end{tabular}

Analysis of the 277 patients eligible for accompanying family suggested that patients who were critically ill and heavily dependent on others for daily life activities had the highest accompanying family rate of $77.55 \%$. This was followed by patients who were critically ill and had positive mental health results, the rate for whom was $62.50 \%$. Patients who were only heavily dependent on others for daily life activities had an accompanying family rate of $50.00 \%$. Patients who were only critically ill had an accompanying family rate of $31.60 \%$. In addition, a total of 69 patients chose to be looked after by two professional escorts, while 95 patients did not have an escort at all.
These results indicate that by assessing the patients' accompanying family needs and managing the escorts carefully, not only are patients' conditions, daily lives, and mental health well looked after, but the number of family escorts are successfully reduced, thereby reducing hospital crowding, ensuring patient safety, and minimizing the frequency of adverse nursing events. Similarly, encouraging patients and their families to hire full-time hospital escorts instead of family escorts is an effective way to control crowding and reduce the risk of virus transmission. 


\section{Discussion}

Over the years, due to historical and cultural background, Chinese people have established family-centered values, resulting in a high accompanying family rate in hospitals. In certain hospitals, this rate exceeds $80 \%[8]$. Hou et al. investigated 357 hospitalized patients and found that their need for escorts was relatively high [9]. Li et al. investigated the reason behind patients' need for accompanying family and found that $58.5 \%$ wanted their family to assist with daily life activities during hospitalization, $68.0 \%$ believed that accompanying family could reflect natural affection, and $78.5 \%$ thought of it as a psychological demand [10]. This is because, due to traditional Chinese culture, patients often experience anxiety, fear, increased dependence, and decreased independence during hospitalization, thereby desiring accompanying family [11]. With the improvement of people's living standards, the pursuit of high-quality medical service, nurses' care, and affection from family has become an essential need of Chinese patients and an important part of Chinese inheritance of rituals, justice, filial piety, and integrity. In the hospital escort mode suitable for the national conditions of our country, the no escort mode is suitable for the special wards such as intensive care unit. However, due to the large number of patients admitted to the general ward and the shortage of nursing human resources, the of no escort mode can not be carried out [12], and the family escort mode has become a common phenomenon. Zhang Jinci, Fan Ying and other [13], survey results show that elderly inpatients have a higher psychological needs for companionship, the vast majority of elderly patients want to be accompanied during hospitalization [14]. During the COVID-19 epidemic, many people, especially critically ill patients and their families, were extremely panicked and concerned about being infected with the virus, thereby demanding greater accompanying family during hospitalization. During the Spring Festival of 2019, the accompanying family rate in the Department of Gastroenterology was as high as $88.67 \%$. Some patients had two or more relatives accompanying them, which compromised the quietness of the ward and increased both the risk of nosocomial infection transmission and nurses' workload in managing the ward, patients, and escorts.

It is important to closely follow the deployment of General Secretary Jinping $\mathrm{Xi}$, implement relevant requirements of provincial, municipal, and district working groups on the prevention and control of COVID-19, and carry out proper protection and control measures in our department, so as to ensure the safety of medical personnel and patients and prevent nosocomial infection transmission. The strict implementation of the accompanying family management system as well as COVID-19 prevention and control measures can improve the mental state and quality of life of patients during hospitalization, thereby promoting their physical and mental recovery. At the same time, it can also reduce the accompanying family rate and minimize hospital crowding, thereby effectively ensuring the safety of medical personnel and escorts and protecting them from being infected with COVID-19. From March to April 2020, since the epidemic was effectively brought under control across China, the state has called on all parts of the country to resume work and production in an orderly manner. In response to this, the First Affiliated Hospital of Jinan University has gradually resumed normal patient diagnosis, treatment, and admission procedures. Although patient numbers have increased, epidemic prevention and control measures should still be strictly followed to prevent infections both within and beyond the hospital. Therefore, it remains important to continue escort management, reduce ward crowding, and prevent infection.

\section{Conclusions}

Establishing and strictly implementing the management system of kinship escort can not only effectively prevent nosocomial infection, but also improve the psychological state of inpatients, improve the quality of life during hospitalization, promote physical and mental recovery, and reduce the rate of accompanying care. Reduce staff density, reduce the risk of iatrogenic infection [15]. It can effectively ensure the safety of medical patients and accompanying people, not infected by new coronavirus, and do a good job of epidemic prevention and control.

\section{References}

[1] General Office of the National Health Commission of the People's Republic of China. Notice on the issuance of novel coronavirus infection of pneumonia diagnosis-804-Chinese Journal of Hospital Infection 2020 Vol. 30 No. 6 Chin J Nosocomial Vol. 30 No. 62020 treatment protocol (trial version of the fifth edition). Notice of the National Health and Family Planning Commission [2020] No. 1032020 [EB/OL]. (2020-02-05)[2020-02-12]

http://www.nhc.gov.cn/yzygj/s7653p/202002/3b09b894ac9b4 204a79db5b8912d4440.shtml.

[2] Bureau of Disease Control and Prevention. Announcement of the National Health Commission of the People's Republic of China: 2020 No. 1 [EB/OL]. (2020-01-20) [2020-02-08]. http://www.nhc.gov.cn/jkj/s7916/202001/44a3b8245e8049d28 $37 \mathrm{a} 4 \mathrm{f} 27529 \mathrm{~cd} 386 . \mathrm{shtml}$.

[3] X. Li, S. Cheng, X. Zhang. "Effect of different types of escorts on the functional recovery and psychological status of spinal cord injury patients". Modern Clinical Nursing, 2008, 7 (10): 5-7.

[4] General Office of the National Health Commission of the People's Republic of China. Notice of the General Office of the National Health Commission on the Issuance of Technical Guidelines for the Prevention and Control of COVID-19 Infections in Medical Institutions (First Edition). [EB/OL]. [2020-1-22].

http://www.chinacdc.cn/jkzt/crb/zl/szkb_11803/jszl_11815/20 2001/t20200123_211392.html.

[5] Xu Meiyu, Mei Yingtai, Lian Zhengmei. [J]] on the Standardized Management and Effect of Occupational Escort in Hospitals. PLA Nursing Journal, 2014, 31 (19): 66, 74. 
[6] Li Zhixian. [J].] Measures to Improve the Quality of Full-time Care Journal of Chinese Medicine Management. February 2016 Vol. 24 No. 3: 63-64.

[7] He Chaogui, Zhou Yuan, Xie Chaoying, etc. Advances in research on unaccompanied wards [J].; and Contemporary Nurses September 2017 (2017) 9-0003-04.

[8] Y. Chen. "Discussion on the status quo and preliminary strategies of patient accompany management in wards". Journal of Qiqihar Medical University, 2003, 24 (6): 675-676.

[9] Z. Hou, R. Li, D. Zeng, et al. "Analysis of the patient's need to be accompanied". Modern Nurse. 2004, 10 (10): 906.

[10] G. Li, M. Xia, X. Yan, et al. "A survey on the primary care and accompany needs of hospitalized patients". Chin. J. Nursing 2011, Vol 17, No. 23: 2754-2755.
[11] B. Zhao. Introduction to Nursing. Beijing: People's Medical Publishing House. 1999: 3-50.

[12] J. Wang, X. Li. "Research progress on the status quo of the hospital accompany model and its influencing factors". J. Nurs. Sci., 2011, 1 (26): 88-90.

[13] Zhang Jinci, Fan Ying. Investigation and Analysis on the Needs of Elderly Inpatients in Care [J]. International Journal of Nursing, 2011, 30 (3): 353-354.

[14] Wang Yali Cui Lijun. Investigation and study on escort needs of inpatients. Chinese Medical Records, vol. 14, No. 2, 2013 34-36.

[15] Teng Xufei. The application value of strengthening escort management in nursing work of infection department. Chinese medicine management records. August 2019 Vol. 27 No. 15 71-72. 